\title{
Pre-immature dendritic cells (PIDC) pulsed with HPV16 E6 or E7 peptide are capable of eliciting specific immune response in patients with advanced cervical cancer
}

\author{
Osama E Rahma ${ }^{1,4}$, Vincent E Herrin ${ }^{1}$, Rami A Ibrahim ${ }^{1}$, Anton Toubaji ${ }^{1}$, Sarah Bernstein ${ }^{2}$, Omar Dakheel ${ }^{1}$, \\ Seth M Steinberg ${ }^{3}$, Rasha Abu Eid ${ }^{5}$, Mikayel Mkrtichyan ${ }^{5}$, Jay A Berzofsky ${ }^{1}$ and Samir N Khleif ${ }^{1,5^{*}}$
}

\begin{abstract}
Background: The protein products of the early genes E6 and E7 in high-risk HPV types 16 and 18 have been implicated in the oncogenic capability of these viruses. Therefore, these peptides represent attractive vaccine therapy targets.

Methods: Thirty-two patients with advanced cervical cancer (HPV16 or 18 positive) were treated with HPV16 E6 (18-26) (Arm A) or HPV16 E7 (12-20) peptide (Arm B) pulsed on PBMCs in order to illicit immune response against the relevant peptide on both arms. These PBMCs were cultured for a short time (48 hours only) and in the presence of GM- CSF, accordingly, they were identified as "Pre-Immature Dentritic Cells".

Results: $51 \mathrm{Cr}$ release assay and ELISPOT demonstrated evidence of specific immune response against the relevant peptide in 10/16 (63\%) evaluable patients in arm A and 7/12 (58\%) in arm B. HPV16 E6 was found to be homologous to HPV18 E6 in both vivo and vitro. The median overall survival (OS) and progression free survival (PFS) for the full cohort was 10.0 and 3.5 months, respectively. There were no RECIST responses in any patient. The majority of toxicities were grade I and II.
\end{abstract}

Conclusions: We demonstrated the feasibility and ability of Pre-Immature Dentritic Cells pulsed with HPV16 E6 (18-26) or HPV16 E7 (12-20) to induce a specific immune response against the relevant peptide despite the advanced disease of the cervical cancer patients treated on this trial. We believe that this observation deserves further investigations.

Keywords: Dendritic cells, HPV16, E6, E7, Vaccine, Cervical cancer

\section{Background}

Cervical cancer is the third most common malignancy in women and the fourth leading cause of female cancer-related death worldwide [1]. More than 500,000 new cases of invasive cervical cancer are diagnosed throughout the world yearly, accounting for over 275,000 deaths [1]. High-risk human papillomavirus (HPV) has been implicated as an etiologic factor in almost all cervical cancers [2]. Current therapeutic strategies for advanced cervical cancer have proven ineffective [3,4].

\footnotetext{
* Correspondence: skhleif@gru.edu

'Cancer Vaccine Branch, CCR, NCl, 10 Center Drive, Bethesda, MD 20892, USA ${ }^{5}$ Georgia Regents University Cancer Center, 1411 Laney Walker Blvd, Augusta, GA 30912, USA

Full list of author information is available at the end of the article
}

The recent development and FDA approval of two vaccines for HPV infection have represented a major advance in cervical cancer prevention [5-8]. However, the effect of prophylactic vaccines on women already infected with HPV remains to be determined, and the impact of prophylactic vaccines on the elimination of cervical cancer is not expected to be apparent for decades [9]. Accordingly, better therapeutic modalities for advanced cervical cancer are needed.

HPV16 and HPV18 are the two most carcinogenic HPV types amongst the high-risk HPV, and they are responsible for about $70 \%$ of invasive cervical cancer cases $[10,11]$. HPV-E6 and E7 are the main oncogenic protein products of high risk viruses and their continuous expression is necessary for the initiation and maintenance

\section{Ciomed Central}

(c) 2014 Rahma et al.; licensee BioMed Central Ltd. This is an Open Access article distributed under the terms of the Creative Commons Attribution License (http://creativecommons.org/licenses/by/4.0), which permits unrestricted use, distribution, and reproduction in any medium, provided the original work is properly credited. The Creative Commons Public Domain Dedication waiver (http://creativecommons.org/publicdomain/zero/1.0/) applies to the data made available in this article, unless otherwise stated. 
of malignant transformation [12-14]. Therefore, these antigens represent attractive targets for therapeutic cancer vaccines.

The methods of peptide vaccine administration have varied. One way of delivery is by pulsing the peptide on dendritic cells (DCs), which are the most potent professional antigen-presenting cells [15-17]. Dendritic cells could be prepared as mature or immature cells. Immature DCs are Peripheral Blood Mononuclear Cells (PBMCs) cultured for 5-7 days in GM-CSF and IL-4. On the other hand, mature DCs are usually cultured with the addition of pro-inflammatory cytokines for an additional 24-48 hours [18,19]. Immature DCs can effectively capture antigens but, as opposed to mature DCs, they lack the full T cell co-stimulatory activity [20]. Therefore, most trials that utilized DCs used the mature type $[17,21,22]$. It is not clear whether maturation of DCs is necessary to generate the needed immune response in cancer vaccines. Furthernore, the preparation of DCs is time consuming, expensive, and requires specialized facilities. Accordingly, here, we tested the feasibility of using PBMCs in a state prior to being immature DCs; "pre immature DCs". PBMCs were cultured for a shorter time (48 hours only) in the presence of GM- CSF only and used to administer peptides E6 (18-26), or E7 (12-20), as cancer vaccines, to patients with advanced cervical cancers. We found that this method of vaccination is well tolerated and capable of eliciting a specific immune response against the relevant peptide.

\section{Methods}

\section{Patient selection}

Patients were assigned to two arms. Arm A received HPV16 E6 (18-26) peptide, while patients on arm B received HPV16 E7 (12-20) peptide pulsed on preimmature dendritic cells (PIDCs). Patients had histologically proven advanced cervical carcinoma, recurrent or persistent disease despite prior treatment, and no evidence of brain metastases. Patients on both arms had HLA.A2.1 subtype. Patients who received the E6 peptide harbored either HPV16 or 18 in their tumors, whereas patients who received the E7 peptide harbored HPV16 (Tables 1 and 2). These selection criteria were based on our pre-clinical studies which showed that HPV16 E6 (18-26) and HPV18 E6 (13-21) peptides are homologous. The sequence of both peptides is identical except for amino acid number 21 where glutamine in HPV16 E6 (18-26) is replaced by aspartic acid in HPV 18 E6 (13-21). All enrolled patients met the protocol eligibility criteria, including ECOG performance status of $0-1$ and life expectancy of more than 3 months. Main exclusion criteria included history of autoimmune disease, and history of other malignancies except basal cell carcinoma of the skin. Both the National Cancer Institute (NCI) and

Table 1 HPV16 E6 patient profile, clinical and immunological outcome

\begin{tabular}{|c|c|c|c|c|c|c|c|c|c|c|c|}
\hline $\mathrm{Pt}$ & Age & Path & HPV type & Disease extension & \# Vac & Off-Study response/reason & PFS (ms) & OS (ms) & $C r-51$ & ELISPOT & $I R$ \\
\hline $1 \mathrm{~A}$ & 39 & $A C A$ & 18 & Mets & 8 & PD & 8.0 & 20.1 & - & + & + \\
\hline $2 \mathrm{~A}$ & 46 & SCC & 16 & Mets & 1 & SD/PPS & $0.7+$ & 1.4 & ND & ND & ND \\
\hline $3 \mathrm{~A}$ & 41 & SCC & 18 & Mets & 6 & PD & 5.9 & 18.6 & - & - & - \\
\hline $4 \mathrm{~A}$ & 37 & SCC & 18 & Mets & 4 & PD & 3.6 & 9.7 & - & + & + \\
\hline $5 A$ & 47 & SCC & 18 & Mets & 2 & SD/PPS & $2.8+$ & 4.9 & - & - & - \\
\hline $6 \mathrm{~A}$ & 36 & ASCC & 16 & Mets & 3 & PD & 1.6 & 8.7 & + & NA & + \\
\hline $7 \mathrm{~A}$ & 40 & ASCC & 18 & Mets & 4 & PD & 4.6 & 6.0 & - & - & - \\
\hline $8 \mathrm{~A}$ & 48 & $A C A$ & 18 & Mets & 4 & PD & 3.4 & 10.0 & - & NA & - \\
\hline $9 A$ & 40 & SCC & 18 & Mets & 1 & SD/PPS & $4.4+$ & 5.2 & ND & ND & ND \\
\hline $10 \mathrm{~A}$ & 43 & SCC & 18 & Mets & 2 & PD & 1.4 & 4.3 & - & + & + \\
\hline $11 \mathrm{~A}$ & 44 & $A C A$ & 18 & Mets & 2 & PD & 1.5 & 4.9 & + & - & + \\
\hline $12 \mathrm{~A}$ & 53 & SCC & 18 & Mets & 2 & PD & 1.6 & 11.1 & - & + & + \\
\hline $13 \mathrm{~A}$ & 36 & SCC & 18 & Mets & 6 & PD & 5.6 & 10.7 & - & + & + \\
\hline $14 \mathrm{~A}$ & 36 & SCC & 18 & NED & 6 & PD & 5.3 & 20.5 & NA & - & - \\
\hline $15 \mathrm{~A}$ & 38 & SCC & 18 & NED & 10 & NED & $67.1+$ & $67.1+$ & - & + & + \\
\hline $16 \mathrm{~A}$ & 36 & SCC & 18 & Mets & 14 & SD & 115.9+ & $115.9+$ & NA & + & + \\
\hline $17 \mathrm{~A}$ & 34 & SCC & 16 & Mets & 4 & PD & 0.4 & 3.9 & NA & + & + \\
\hline $18 \mathrm{~A}$ & 41 & SCC & 16 & Mets & 3 & PD & 1.5 & 3.7 & NA & - & - \\
\hline
\end{tabular}

Abbreviations: Pt Patient, Path Pathology, \#Vac number of administered vaccines, IR Immune Response, ND Not done, NA Not available, Cr-51 Chromium-51 release assay, ELISPOT Enzyme-Linked ImmunoSpot, SCC Squamous Cell Carcinoma, ACA Adenocarcinoma, ASCC Adenosquamous Carcinoma, NED No Evidence of Disease. PD Progression of Disease, PPS Poor Performance Status, SD Stable Disease, Mets metastatic disease, PFS Progression Free Survival, OS Overall Survival, ms Months. 
Table 2 HPV16 E7 Patient profile, clinical and immmunological outcome

\begin{tabular}{|c|c|c|c|c|c|c|c|c|c|c|c|}
\hline$\overline{\mathrm{Pt}}$ & Age & Path & HPV type & Disease extension & $\#$ \#ac & Off-Study response/reason & PFS (ms) & OS (ms) & $C r-51$ & ELISPOT & $I R$ \\
\hline $1 \mathrm{~B}$ & 37 & ACA & 16 & Mets & 9 & PD & 10.0 & 25.2 & + & N/A & + \\
\hline $2 B$ & 40 & SSC & 16 & Mets & 5 & $\mathrm{PD}$ & 6.3 & 19.9 & + & N/A & + \\
\hline $3 B$ & 41 & SSC & 16 & Pelvis & 4 & PD & 4.6 & 8.5 & + & + & + \\
\hline $4 \mathrm{~B}$ & 33 & SSC & 16 & Pelvis & 2 & PD & 2.4 & 13.9 & - & NA & - \\
\hline $5 B$ & 53 & SSC & 16 & Mets & 4 & PD & 1.6 & 7.2 & - & + & + \\
\hline $6 B$ & 37 & SSC & 16 & Pelvis & 1 & PD & 1.5 & 4.4 & ND & ND & ND \\
\hline $7 B$ & 48 & SSC & 16 & Mets & 4 & PD & 1.7 & 6.3 & - & - & - \\
\hline $8 B$ & 53 & SSC & 16 & Mets & 4 & PD & 3.6 & 42.6 & - & - & - \\
\hline $9 B$ & 37 & SSC & 16 & Pelvis & 6 & PD & 5.3 & 7.4 & - & + & + \\
\hline $10 \mathrm{~B}$ & 68 & SSC & 16 & Pelvis & 3 & PD & 2.5 & 5.5 & - & - & - \\
\hline $11 \mathrm{~B}$ & 49 & SSC & 16 & Mets & 8 & PD & 7.7 & $7.7+$ & + & + & + \\
\hline $12 \mathrm{~B}$ & 51 & SSC & 16 & Mets & 2 & PD & 1.6 & 18.4 & - & - & - \\
\hline $13 B$ & 45 & SSC & 16 & Mets & 6 & $\mathrm{SD} / \mathrm{W}$ & $7.5+$ & $7.5+$ & - & + & + \\
\hline $14 \mathrm{~B}$ & 28 & SSC & 16 & Mets & 4 & PD & 3.5 & 11.5 & NA & NA & NA \\
\hline
\end{tabular}

Abbreviations: Pt Patient, Path Pathology, \#Vac number of administered vaccines, IR Immune Response, ND Not done, NA Not available, Cr-51 Chromium-51 release assay, ELISPOT Enzyme-Linked ImmunoSpot, SCC Squamous Cell Carcinoma, ACA Adenocarcinoma, ASCC Adenosquamous Carcinoma, NED No Evidence of Disease. PD Progression of Disease, PPS Poor Performance Status, SD Stable Disease, Mets metastatic disease, PFS Progression Free Survival, OS Overall Survival, ms Months.

National Naval Medical Center (NNMC) Institutional Review Boards (IRBs) approved the protocol and the patients' consent was obtained prior to enrollment.

\section{Peptide selection}

Using computer-based prediction web sites such as the syfpeithi [(http://www.syfpeithi.de/Scripts/MHCServer.dll/ EpitopePrediction.htm)] and the Bimas [(http://thr.cit.nih. gov/molbio/hla_bind/)], the HLA-A2 restricted epitopes in the E6 and E7 sequence were detected. E6 (18-26) peptide (PR-KLPQLCTEL-LYS-9-LEU) and E7 (12-20) peptide (MLDLQPETT-MET-9-THR) were the strongest A2 binders. Therefore, they were used in this trial.

\section{Vaccine preparation}

Pre-immature Dendritic cells (PIDCs) were prepared from peripheral blood mononuclear cells (MNCs) obtained by either automated leukapheresis (Aph) or whole blood (WB) collection. The sequence of MNC collection for vaccine preparation in each patient was Aph, Aph, WB, WB, with repetition of this sequence for subsequent cycles. For Aph cycles, patients underwent leukapheresis on the Fenwal CS3000 blood cell separator using anticoagulation with ACA-A and peripheral or central venous access, with a volume processed of 3-5 liters. MNCs were purified from the leukapheresis collection by an automated ficoll-hypaque density gradient procedure, and $2 \times 10^{9}$ cells were placed into culture for 2 days with RPMI 1640 (Cambrex, Walkersville, MD) supplemented with GM-CSF, $0.4 \mathrm{ng} / \mathrm{ml}$, and $5 \%$ autologous heatinactivated plasma in $162 \mathrm{~cm}^{2}$ flasks (Costar, Myriad,
San Diego, CA), at $37^{\circ} \mathrm{C}$, with $5 \% \mathrm{CO} 2$. After $40-46$ hours, cells were concentrated to $2 \times 10^{7} / \mathrm{ml}$, and $1 \times 10^{9}$ cells were pulsed with the HPV16 E6(18-26) peptide (PRKLPQLCTEL-LYS-9-LEU) or HPV16 E7 (12-20) peptide (MLDLQPETT-MET-9-THR), $10 \mu$ molar, for 2 hours, washed, and resuspended in PlasmaLyte A with $2 \%$ autologous plasma or serum to a volume of $25-40 \mathrm{ml}$, and irradiated with 25 Gy from a Cesium source. For WB cycles, $100-150 \mathrm{ml}$ of peripheral venous blood was drawn into heparin sulfate (10 units $/ \mathrm{ml}$ blood). MNCs were separated on a ficoll hypaque gradient, washed and resuspended. $70 \times 10^{6}$ cells were cultured at a concentration of $4-5 \times 10^{6} / \mathrm{ml}$ in RPMI 1640 (Cambrex) with $5 \%$ heatinactivated autologous serum or allogeneic $A B$ human serum, supplemented with $0.4 \mathrm{ng} / \mathrm{ml}$ of GM-CSF at $37^{\circ} \mathrm{C}$ with 5\% CO2. The HPV16 E6 peptide, $10 \mu$ molar, was added at initiation of culture, and was therefore present for the entire 2 day culture duration. After 40-46 hours, the peptide-pulsed cells were harvested, washed, and resuspended in PlasmaLyte A with $2 \%$ autologous serum or allogeneic $A B$ serum to a volume of $6 \mathrm{ml}$, and irradiated with 25 Gy. For Aph cycles, infusion of up to $1 \times 10^{9}$ peptide-pulsed PIDCs was allowed, with a cell concentration of $20 \times 10^{6} / \mathrm{ml}$, whereas the WB cycles allowed a maximum of $70 \times 10^{6}$ cells, with a cell concentration of $10 \times 10^{6} / \mathrm{ml}$.

\section{Vaccine administration}

Prior to vaccine administration, peptide hypersensitivity testing was done. Briefly, $1 \mu \mathrm{g}$ of peptide dissolved in $0.1 \mathrm{ml}$ of preservative-free normal saline was injected 
intradermally in the outpatient clinic, the patient was observed for 1 hour. The peptide-pulsed PIDCs were infused intravenously (IV) at a rate of $5 \mathrm{ml}$ over 1-2 min through a sterile 110 micron filter needle. Vaccination was repeated at week 3 , then every 4 weeks for a total of 14 vaccinations or until disease progression. Patients were observed for another hour after vaccination.

\section{Clinical monitoring}

Patients were evaluated for toxicity and tumor response during treatment and up to two years after the last vaccination. Tumor response was assessed by physical exam and by the appropriate imaging technique (CT-scan) according to RECIST criteria at baseline, then every 2 vaccinations during therapy and every 3 months during follow-up. Patients were taken off protocol because of either deterioration in the performance status, disease progression or withdrawal from the study. Disease progression was defined per the modified WHO criteria of progression as the appearance of new lesions and/or a $25 \%$ increase of measurable lesions as evident by CT-scan. Once patients had progressed, follow-up was not required except to document late toxicities and death. Adverse events and toxicities were defined and graded according to the NCI Common Toxicity Criteria.

\section{Immunological testing}

To assess the immune response, lymphocytes were isolated from PBMC. Immunologic testing was performed using Chromium-51 release assay (51Cr release assay) against cervical cancer cell lines as described below and Enzyme-Linked ImmunoSpot (ELISPOT). The postvaccination samples were tested at the date of the next administered vaccines prior to each vaccination and compared to pre-vaccination samples. The prevaccination and post-vaccination samples were frozen and assayed at the same time. The immune response was considered positive for patients who demonstrated a positive immune response by either ELISPOT, $51 \mathrm{Cr}$ release assay or both.

\section{Cell lines}

Cervical cancer cell lines used in this trial were either from the ATCC (Mannassas, VA) including the ME180, Caski and MS751 or established at the NCI (Bethesda, $\mathrm{MD})$ like the Cav cervical cancer cell line. All cells were tested in our lab for HLA and HPV typing. Caski, MS751 and Cav are all HLA-A2 positive cell line while ME180 is an A2 negative cell line. Caski harbors the HPV16 genome while MS751 and the Cav harbor the HPV18 genome (low and high number of copies respectively). All cells grew in RPMI supplemented with 10\% FCS, Penicillin, L-Glutamine and Sodium Pyruvate.

\section{Chromium-51 release assay $(51 \mathrm{Cr}$ release assay)}

The cervical cancer cell lines mentioned above were used as targets in the cytotoxic $\mathrm{T}$ lymphocyte (CTL) assay. The cytolytic activity was measured by using the 4-hour $51 \mathrm{Cr}$ release assay. Lymphocytes were prepared from PBMC. These cells were selected and expanded in vitro in the presence of 1-20 $\mu \mathrm{M}$ of E6 peptide with $5 \mathrm{IU} / \mathrm{ml}$ of IL-2 (Chiron, Emeryville, CA) added on day 3 for 7 days. This 7 day cycle was repeated one more time, after which the cells were harvested and tested for specific cytolytic activity against E6 or E7 peptide pulsed autologous APC's (EBV transformed B cells) or tumor cell lines harboring the HPV genome. Target cells were washed with RPMI medium and labeled with $200 \mu \mathrm{Ci}$ ${ }^{51} \mathrm{Cr}$ sodium dichromate in the presence or absence of $10 \mu \mathrm{M}$ of E6 or E7 peptide for 2 hours, after which target cells were washed and plated in 96 well round bottomed plates. Effectors were added to labeled targets at the desired effector to target ratio. The plates were centrifuged at $500 \mathrm{rpm}$ for $5 \mathrm{~min}$., and incubated at $37^{\circ} \mathrm{C}$ for 4 hours. Supernatants were harvested using a Skatron supernatant harvesting device, and the samples counted on a gamma counter. The percent specific lysis was determined, on triplicate samples, by the following formula:

$$
\begin{aligned}
\text { Percent specific lysis }= & \text { experimental release } \\
& - \text { spontaneous release } \times 100
\end{aligned}
$$

\section{Maximum release - spontaneous release}

A result was considered positive with a two fold increase or more in lytic units pre-immunization. If there was no detectable pre-immunization specific lysis, a post immunization lysis of greater than $10 \%$ above the nonpeptide was considered positive, with an Effector:Target (E:T) ratio specified at 50:1. If an E:T ratio of 50:1 was not tested, the next highest ratio was used. In some patients we established CTL and tumor cell lines for future use in studies of antigen specificity.

\section{Enzyme-linked ImmunoSpot (ELISPOT) assay}

ELISPOT assays were performed at the Laboratory of CellMediated Immunity, SAIC-Frederick (CLIA-certified lab). Two frozen normal donor controls with known responsive values were run with each assay to assure quality control of the assay results. For all assays, at least one of the two controls was within 2 standard deviations of the laboratory-generated means for CMV and CEF. All assays were performed on 7-8 day in vitro stimulated PBMCs $(100 \mathrm{~K} /$ well) as the effectors and peptide-pulsed autologous PBMCs $(100 \mathrm{~K} /$ well $)$ as the antigen presenting cells (APCs). When possible, PBMCs from the earliest time point were used as the APCs. However, if this 
was not possible, the pulsed PBMCs were assayed alone to make sure they were not producing any spots. Briefly, the day before assay setup, 96-well polyvinylidene fluoride (PVDF) membrane, HTS opaque plates (Millipore, Billerica, Massachusetts, MSIPS4W10) were coated overnight with a 1:100 dilution of anti-human IFN- $\gamma$ capture antibody $(1 \mathrm{mg} / \mathrm{mL}$, Mabtech Inc., Mariemont, $\mathrm{OH}$, Cat\# 3420-3-1000) in Dulbecco's phosphate buffered saline (DPBS) at room temperature. Antibody-coated plates were washed four times in DPBS the next day and blocked with 5\% human AB ELISPOT medium at $37^{\circ} \mathrm{C}$ for approximately 2 hours. $1 \times 105$ in vitro stimulated PBMCs and $1 \times 105$ autologous, peptide-pulsed PBMCs were plated per well. The plates were incubated for $18-20$ hours at $37^{\circ} \mathrm{C}$. The next day, the plates were manually washed six times with $0.05 \%$ Tween 20 in DPBS, followed by a 2-hour incubation at room temperature with a 1:2000 dilution of the biotinylated secondary antibody, anti-human IFN- $\gamma(1 \mathrm{mg} / \mathrm{mL}$ Mabtech Inc., Mariemont, OH, Cat\# 3420-6-1000) in DPBS/1\% bovine serum albumin $/ 0.05 \%$ Tween. After incubation and four washes in DPBS to remove excess antibody, a 1:3000 dilution of streptavidin alkaline phosphatase (Mabtech, Mariemont, OH, Cat\# 3310-10) in DPBS/1\% bovine serum albumin, was added to each well for 1 hour at room temperature followed by 4 manual washes in DPBS. Finally, the BCIP/NPT substrate, $100 \mu \mathrm{l} /$ well, (KPL, Gaithersburg, Maryland, Cat\# 50-81-08) was added for 7-10 minutes, resulting in the development of spots. The reaction was stopped by washing three times in distilled water. Plates were dried overnight and the spots were visualized and counted using the ImmunoSpot Imaging Analyzer system (Cellular Technology Ltd., Cleveland, OH). ELISPOT results were expressed as the "number of spots per $10^{6}$ responder cells" after subtracting background spots obtained in wells of effectors with nonpulsed PBMCs. For each subject, PBMCs obtained before and after vaccination were analyzed in the same assay to avoid inter-assay variability. A post-immunization fold increase $>2$ compared to pre-immunization was considered a positive response.

\section{Statistical analysis}

This study was carried out as a pilot trial to gain experience administering vaccines with the E6 and E7 peptide. The primary objectives of this study was to determine whether vaccinating advanced cervical cancer patients with Pre-Immature Dendritic Cells (PIDC) pulsed with HPV16 E6 or E7 peptide is safe and capable of eliciting specific immune response against the relevant administered peptide. It was intentionally small in order to have adequate numbers for evaluation of feasibility and immune response, but limited information on clinical efficacy. All results presented are intended to be hypothesis generating in view of the intention of the trial and the limited number of subjects treated.

As secondary outcomes from the trial, overall survival survival (OS) and progression free survival (PFS) were computed from the date the consent was signed until the date the patient died, progressed or was last followed as appropriate. The Kaplan-Meier method was used to estimate the probability of survival or progression free survival as a function of time.

\section{Results}

\section{Patient profile}

Thirty-two patients with advanced cervical cancer were enrolled on this trial. Eighteen patients received the HPV1A6 E6 (18-26) peptide ( $\mathrm{arm} \mathrm{A}$ ) and 14 patients received the HPV16 E7 (12-20) peptide (arm B). The patients' characteristics are summarized in Table 1 (Arm A) and Table 2 (Arm B). Age ranged from 28 to 68 years with a mean of 40.8 in arm A and 44.2 in arm B. Twenty six patients had squamous cell carcinoma (13 in each arm), 4 patients had adenocarcinoma (3 in arm A and 1 in $\operatorname{arm} B$ ), and 2 patients in arm A had adeno-squamous carcinoma. In arm $\mathrm{A}$, four patients' tumors harbored HPV16 and fourteen patients' tumors harbored HPV18. Whereas in $\operatorname{arm} \mathrm{B}$, all patients' tumors harbored the HPV16 subtype. Patients in both arms were heavily pretreated; Twenty two patients underwent at least one surgery with a course of radiation. Two patients received at least one surgery with a course of chemotherapy. The remaining eight patients received only radiation and chemotherapy. Patients had measurable disease on enrollment except patients $14 \mathrm{~A}$ and $15 \mathrm{~A}$ who had no evidence of disease. Patient 14A had stage IB on diagnosis which was treated with surgery followed by adjuvant radiation therapy. Subsequently, further treatment with radiation therapy and chemotherapy was given for recurrent disease in the para-aortic lymph nodes. Patient 15A was diagnosed with stage IIB during laparotomy, and treated with adjuvant radiation therapy. However, this patient developed pulmonary metastasis and was treated with radiation and chemotherapy followed by thoracotomy.

\section{Immunological data}

Phenotype of the pre-immature dendritic cells (PIDCs)

FACS analysis was done on the PIDCs before vaccine administration to document their phenotype. The data was further confirmed using PBMCs from healthy donors. Prior to the GM-CSF incubation; the PIDCs were all monocytes expressing CD14 and they did not express any of the antigen presenting cell surface markers. After two days in culture in the presence of GM-CSF, and pulsing for two hours with the HPV16 E6 or E7 peptide, in spite of the persistent expression of CD14 at slightly lower levels (Figure 1A), CD14 positive 

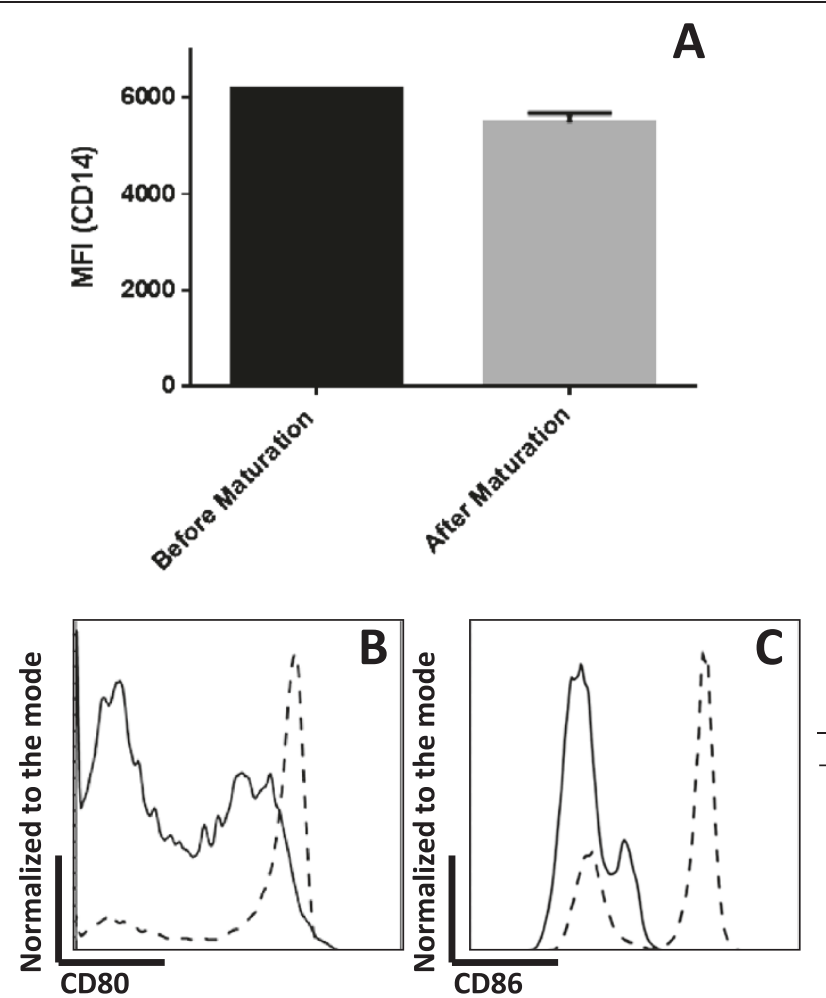

PBMC Oh
- PBMC 48h + peptide
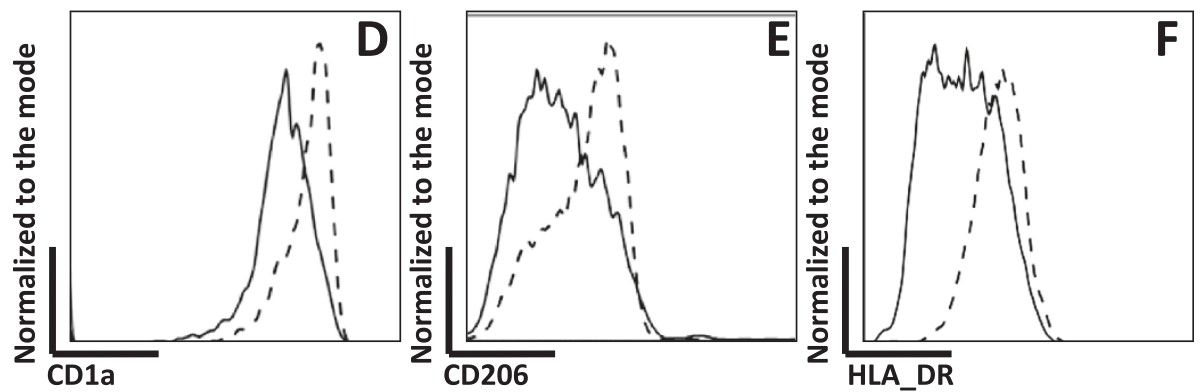

Figure 1 FACS analysis showing the phenotype of PIDCs used in the vaccine preparation. A) After a two day incubation in the presence of GM-CSF, and pulsating with HPV E7 peptide for two hours, CD14 positive cells persistently expressed CD14 at a slightly lower level. B) CD14 positive cells expressed a higher level of CD80 following incubation with GM-CSF and HPV E7 peptide. C) CD14 positive cells expressed a higher level of CD86 following incubation with GM-CSF and HPV E7 peptide. D) CD14 positive cells expressed a higher level of CD1a following incubation with GM-CSF and HPV E7 peptide. E) CD14 positive cells expressed a higher level of the Mannose receptor (CD206) following incubation with GM-CSF and HPV E7 peptide. F) HLA-DR was up-regulated in CD14 positive cells following incubation with GM-CSF and HPV E7 peptide.

cells (both from PIDCs and PBMCs) were found to express antigen presenting cell associated surface markers (CD80, CD86, CD1a and Mannose receptor (CD206)) (Figure 1B-E). Furthermore, there was up regulation in HLA-DR (Figure 1F).

\section{The cross reactivity between HPV16 E6 and HPV18 E6}

To test whether E6 protein is processed and presented in the proper context of MHC class I and to test the ability of T-cells generated by HPV16 E6 to cross react with an internally processed HPV18 E6, we tested the ability of post vaccination PBMCs to lyse established HLA-A2 positive cervical cancer cell lines harboring either the HPV16 or HPV18 genome. We found that these primed T-cells were able to lyse Caski cells (HLA-A2 and HPV16 positive), Cav cell line (HLA-A2 with a high number of copies of HPV18) and, to a lesser extent, the MS751 cell line (HLA-A2 harboring lower number of copies of HPV18) but not the HLA-A2 and HPV negative cervical cancer cells (ME180). We were able to see specific lysis in those cell lines and the lysis was higher in cell lines that carry higher number of copies of HPV (Figure 2). Similarly, the effector cells generated through the E7 vaccine were tested against tumor cells either expressing the HPV16 E7 genome or HPV16 E7 negative cell line. We were able to detect 


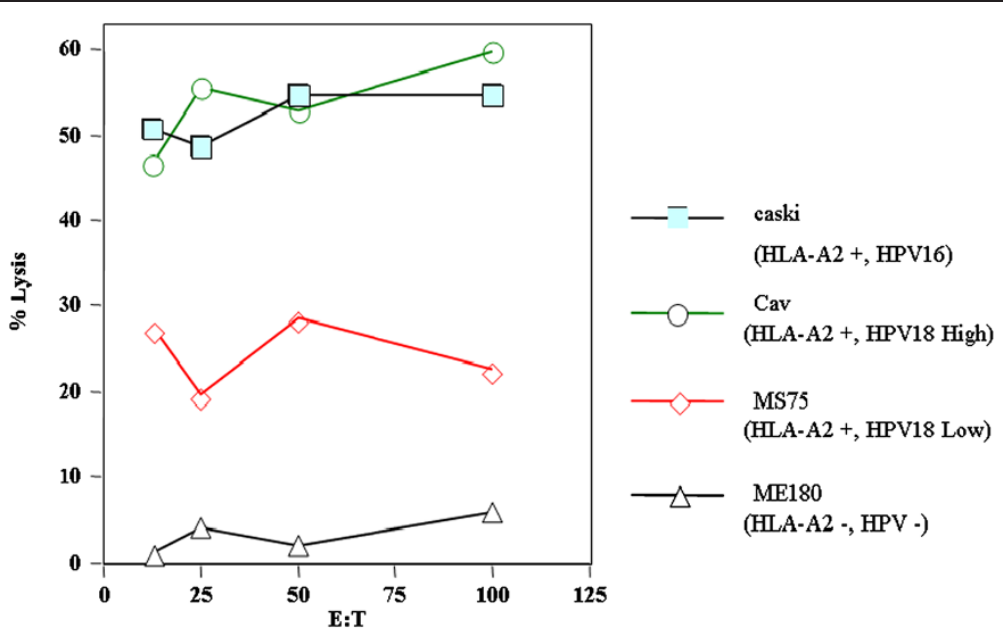

Figure 2 The cytolytic activity of patient $6 A^{\prime}$ PBMCs post one vaccination. Cervical cancer cell lines were used as target cells. Caski (HPV16 positive, HLA-A2 cell line) closed squares; CAV (HPV 18 high and HLA-A2 cell line), open circles; MS751 (an HPV 18 low, HLA-A2 cell line), open diamonds; ME180 (HPV negative, HLA-A2 negative cervical cancer cell line), open triangles. Highest percentage of specific lysis was seen with the Caski and Cav cell lines, followed by the MS751 and only background level lysis was seen when the ME180 cell line was used as a target.

lysis of the E7 positive cell lines and not the E7 negative ones. Furthermore, effector cells were able to lyse only the HLA-A2 positive cell lines and not the HLAA2 negative ones (data not shown).

\section{Immune response}

Immunological responses were evaluated in 28 out of 32 treated patients (Tables 1 and 2). Three patients were excluded from the analysis since they received only one vaccine due to disease progression $(2 \mathrm{~A}, 9 \mathrm{~A}, 6 \mathrm{~B})$, and one patient (14B) was not tested for an immune response due to lack of sufficient cell numbers to reproduce the results. Seventeen out of the 28 (61\%) evaluated patients had positive immune responses by either ELISPOT, $51 \mathrm{Cr}$ release assay, or both $[10 / 16(63 \%)$ in arm A, and $7 / 12(58 \%)$ in arm B]. The immune response data for both ELISPOT and $51 \mathrm{Cr}$ release assay are presented in Additional file 1: Figures S1 and Additional file 2: Figure S2.

\section{Clinical response}

Clinical response was evaluated in 29 out of the 32 treated patients (Tables 1 and 2). Three patients (2A, 9A and $6 \mathrm{~B}$ ) were excluded from the analysis since they received only one vaccine due to poor performance status. Out of the 29 evaluable patients the clinical outcome was as follows: Twenty five patients had progression of disease during the course of treatment (13/16 on arm A and $12 / 13$ patients on arm B); one patient (5A) was taken off the study due to poor performance status after receiving 2 vaccine doses; one patient (\#13B) decided to withdraw from the study and had a stable disease after receiving 6 vaccine doses; and the remaining 2 patients
(\#15A and 16A) completed the treatment without disease progression. Patient 15A was enrolled on the study with NED after being treated with 2 surgeries, 2 courses of radiation therapy and one chemotherapy regimen. This patient received a total of 10 vaccine doses and continued to have no evidence of disease as of the last follow up (67.1 months). Patient 16A was enrolled on the study with stage IV disease after being treated with radiation therapy and 2 courses of chemotherapy. This patient completed a total of 14 vaccine doses and continued to have a stable disease, as of the last available follow up (115.9 months). There were no RECIST responses in any patient. The median overall survival (OS) and progression free survival (PFS) for the full cohort was 10.0 and 3.5 months, respectively (Figure 3 ). Arm A had a median OS and PFS of 9.7 and 3.6 months, respectively, while patients on arm B had a median OS and PFS of 11.4 and 3.5 months, respectively. Eighteen patients received fewer than five vaccine doses and had a median OS and PFS of 7.2 and 1.7 months, respectively. On the other hand, 11 patients who were able to receive five or more vaccine doses had a median OS and PFS of 20.1 and 6.9 months, respectively. Patients who had an immune response had a median OS and PFS of 8.7and 5.3 months, respectively, while the nonimmune responders had a median OS and PFS of 6.3 and 2.8 months, respectively.

\section{Safety and toxicity}

The vaccine was well tolerated. No allergic reaction to the vaccine was reported in either arm. The majority of toxicities were grade I or II, with fatigue being the most common occurring in 12/18 (67\%) patients in arm A 

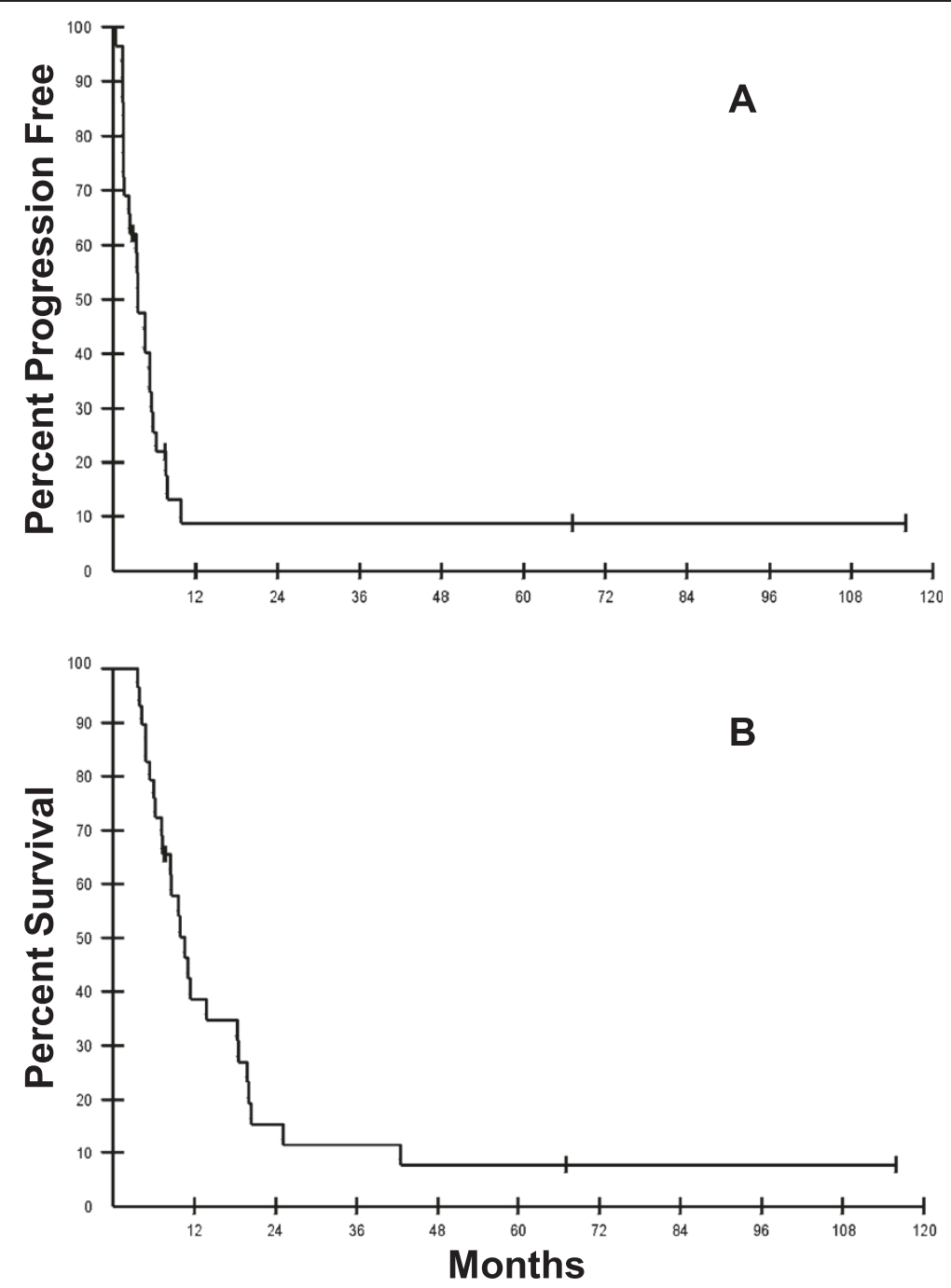

Figure 3 Progression free survival and overall survival for the full cohort. Progression free survival (A) and overall survival (B) for the full cohort. PFS was calculated as time from the date of consent until evidence of disease progression or last follow-up. OS was calculated as time from consent date until death or last follow-up.

and 6/14 (43\%) of patients in arm B. Grade III toxicities were reported in both arms, majority of which were attributed to the disease process. These events included: anemia, nausea, vomiting, shortness of breath, chest pain, pyelonephritis, and thrombocytopenia. No grade IV toxicity was reported on either arm (Table 3 ).

\section{Discussion}

In this pilot study Pre-Immature Dentritic Cells (PIDC) pulsed with HPV16 E6 (18-26) or HPV16 E7 (12-20) peptides were found to be capable of generating specific immune response as evident by the $51 \mathrm{Cr}$ release CTL or ELISPOT assay. The majority of patients on both arms had a measurable increase in the number of IFN- $\gamma$ secreting cells post vaccination. In the cytolytic assay, we were able to show that PBMCs of patients who received the vaccine were able to recognize and lyse target cells pulsed with the E6 or E7 peptide. In addition, we showed that the HPV16 E6 is homologous to HPV18 E6 and crossreact. We were able to demonstrate that the immune response induced by HPV16 E6 can specifically lyse cervical cancer cell lines harboring either HPV16 (caski cell line) or HPV18 (Cav and MS751) in vitro. These findings were also evident in ex vivo since patients in the E6 arm developed an immune response against HPV16 E6 peptide whether they expressed HPV16 or 18 on their tumors. Accordingly, since those two peptides are able to cross react either of them can be used for vaccination of both groups of patients with HPV16 and HPV 18 expressing tumors.

The antigen presenting cells used in this trial were autologous PBMCs cultured only for 48 hours in the presence of GM-CSF, and hence we called them 
Table 3 Toxicities

\begin{tabular}{ll}
\hline Toxicity grade $\mathbf{3}$ & \# of patients \\
\hline Anemia & $1(13 \mathrm{~A})$ \\
Nausea & $1(17 \mathrm{~A})$ \\
Vomiting & $1(17 \mathrm{~A})$ \\
SOB & $1(18 \mathrm{~A})$ \\
Chest pain & $1(18 \mathrm{~A})$ \\
Pyelonephritis & $1(9 \mathrm{~A})$ \\
Thrombocytopenia & $1(14 \mathrm{~B})$ \\
\hline
\end{tabular}

Abbreviations: $S O B$ shortness of breath.

"Pre-Immature Dendritic Cells (PIDCs)". In our trial, $61 \%$ of patients developed immune response against the relevant peptide indicating that the PIDCs were able to function normally and proved to be potent APCs, even though they don't express all surface markers of DCs. These PIDCs possess a great advantage over regular DCs as they can be generated in a shorter time, can give a higher cell yield, and are not dependent on the cytokines in the culture such as IL-4.

Two previously conducted clinical trials demonstrated the immunogenicity of the recombinant E7 protein pulsed on mature DCs in advanced cervical cancer patients $[21,22]$. Ferrara et al. treated patients with recombinant HPV16 E7 or HPV18 E7 protein pulsed on mature DCs and administered subcutaneously and demonstrated the generation of immune response in 3 of the 11 treated patients $(27 \%)$ [21]. Santin et al. treated patients with recombinant HPV16 E7 or HPV18 E7 protein pulsed on mature DCs and administered subcutaneously in combination with low dose IL-2 and showed positive immune responses in all tested patients $(4 / 4,100 \%)$ [22]. A live recombinant vaccinia virus expressing the E6 and E7 proteins of HPV16 and 18 was also investigated as a therapeutic vaccine in the advanced cervical cancer setting [23]. Only 1/3 (33\%) of evaluated patients had a positive CTL response following re-stimulation with E6 and E7 peptides. More recently, Melief et al. investigated a mix of long peptides from the HPV-16 E6 and E7 in incomplete Freund's adjuvant in 20 patients with HPV16 positive, high-grade vulvar intraepithelial neoplasia. Fifteen of 19 patients (79\%) had clinical responses and all patients had vaccine-induced T-cell responses [24]. Indeed, it would be difficult to compare our findings to others given the different methods of vaccination and the variety of immune assays used to detect the immune response.

Although this trial was not powered to test for clinical efficacy, it revealed some interesting clinical findings. For the full cohort, the median overall survival (OS) and progression free survival (PFS) was 10.0 and 3.5 months, respectively which is encouraging given the poor expected survival in this population of patients when treated with standard chemotherapy (median OS of 4.9 months) [3]. Moreover, it is noticeable that patients who received five or more vaccine doses had a longer median OS and PFS of 20.1 and 6.9 months, respectively. Because this is not a randomized study, it would be difficult to determine whether the longer survival in this group of patients is due the higher number of vaccine doses they received or to their tumors' biology which allowed them to survive longer and therefore to receive more vaccines. Furthermore, the disconnection between the immune and the clinical response could be related to the expansion of inhibitory immune cells such as T-regulatory cells (T-regs) and myeloid-derived suppressor cells (MDSCs) or the activation of other immunosuppressive pathways such as the PD-L1/PD-1 pathway. However, because of the small cohorts tested on this trial we cannot conclude a relationship between OS or PFS and immune response.

In summary, this pilot study represents a proof of concept for using pre-immature dendritic cells (PIDCs) for peptide delivery in cancer vaccine development. This method should be further investigated in future clinical trials. In addition, future directions should focus on investigating this method of peptide vaccination in a population of patients with early stage of disease in order to allow time for the generated immune response to be translated to a clinical effect. Combining this method of vaccination with other modalities such as chemotherapy, radiation therapy or immune modulators should be also considered in the future.

\section{Conclusion}

In summary, our trial confirmed the feasibility and safety of using HPV16 E6 and E7 peptide vaccine as a personalized treatment for patients with advanced cervical cancer. These peptide vaccines were capable of generating a specific immune response against the relevant peptide. Further studies are needed to test these peptide vaccines in early disease setting and in combination with other modalities such as immune modulators.

\section{Additional files}

\footnotetext{
Additional file 1: Figure S1. Immune responses in patients on arm A (HPV16 E6). Immune responses of patients on arm A to HPV16E6 peptide in red compared with no peptide in blue. Immune responses were measured by ELISPOT (Panel A) or 51Cr release assay (Panel B). Abbreviations: Pre-vaccine, Pre-vaccination sample; Post- $V$, Post-vaccination sample marked by the vaccine number; $f / u$, Follow up sample marked in months (ms) from the last post vaccine sample.

Additional file 2: Figure S2. Immune responses in patients on arm B (HPV16 E7). Immune responses of patients on arm B to HPV16E7 peptide in red compared with no peptide in blue. Immune responses were measured by ELISPOT (Panel A) or 51Cr release assay (Panel B). Abbreviations: Pre-vaccine, Pre-vaccination sample; Post- $V$, Post-vaccination sample marked by the vaccine number; f/u, Follow up sample marked in months (ms) from the last post vaccine sample.
} 


\section{Abbreviations}

PIDC: Pre-immature dendritic cells; HPV: Human papilloma virus; PBMCs: Peripheral blood mononuclear cell; GM- CSF: Granulocyte macrophage colony-stimulating factor; $51 \mathrm{Cr}$ release: Chromium-51 release assay; ELISPOT: Enzyme-linked ImmunoSpot; OS: Overall survival; PFS: Progression free survival; RECIST: Response evaluation criteria; DCs: Dendritic cells; IL: Interleukin; ECOG: Eastern cooperative oncology group; NCl: National cancer institute; NNMC: National naval medical center; IRBs: Institutional review boards; MNCs: Peripheral blood mononuclear cells; Aph: Automated leukapheresis; WB: Whole blood; IV: Intravenously (IV); CTL: Cytotoxic T Lymphocyte; TLN: Tumor-draining lymph nodes; APC's: Antigen presenting cells; IFN-gamma: Interferon-gamma.

\section{Competing interests}

The authors declare that they have no competing interests.

\section{Authors' contributions}

OER collected and analyzed the data and drafted the manuscript. VEH participated in patient care, evaluated patients for eligibility, safety endpoints, and efficacy including follow-up assessments. RAI, AT, RAE, and MM performed the experimental assays. OD collected the data. SB participated in patient care. SMS performed the statistical analysis. JAB participated in the design of the study. SNK conceived of the study and participated in its design and coordination. All authors read and approved the final manuscript.

\section{Source of funding}

This research was supported by the Intramural Research Program of the $\mathrm{NIH}$, National Cancer Institute, Center for Cancer Research. The content of this publication does not necessarily reflect the views or policies of the Department of Health and Human Services, nor does mention of trade names, commercial products, or organizations imply endorsement by the US Government.

\section{Author details}

${ }^{1}$ Cancer Vaccine Branch, CCR, NCl, 10 Center Drive, Bethesda, MD 20892, USA. ${ }^{2}$ Walter Reed National Military Medical Center, 8901 Wisconsin Ave, Bethesda, MD 20814, USA. ${ }^{3}$ Biostatistics and Data Management Section, CCR, NCl, 9609 Medical Center Drive, Rockville, MD 20850, USA. ${ }^{4}$ University of Virginia, Charlottesville, VA 22908, USA. ${ }^{5}$ Georgia Regents University Cancer Center, 1411 Laney Walker Blvd, Augusta, GA 30912, USA.

Received: 9 October 2014 Accepted: 2 December 2014

Published online: 16 December 2014

\section{References}

1. Jemal A, Bray F, Center MM, Ferlay J, Ward E, Forman D: Global cancer statistics. CA Cancer J Clin 2011, 61(2):69-90.

2. Walboomers JM, Jacobs MV, Manos MM, Bosch FX, Kummer JA, Shah KV Snijders PJ, Peto J, Meijer CJ, Munoz N: Human papillomavirus is a necessary cause of invasive cervical cancer worldwide. J Pathol 1999, 189(1):12-19.

3. Moore DH, Blessing JA, McQuellon RP, Thaler HT, Cella D, Benda J, Miller DS, Olt G, King S, Boggess JF: Phase III study of cisplatin with or without paclitaxel in stage IVB, recurrent, or persistent squamous cell carcinoma of the cervix: a gynecologic oncology group study. J Clin Oncol 2004 22(15):3113-3119.

4. Long HJ 3rd, Bundy BN, Grendys EC Jr, Benda JA, McMeekin DS, Sorosky J, Miller DS, Eaton LA, Fiorica JV: Randomized phase III trial of cisplatin with or without topotecan in carcinoma of the uterine cervix: a gynecologic oncology group study. J Clin Oncol 2005, 23(21):4626-4633.

5. Villa LL, Costa RL, Petta CA, Andrade RP, Ault KA, Giuliano AR, Wheeler CM, Koutsky LA, Malm C, Lehtinen M, Skjeldestad FE, Olsson SE, Steinwall M, Brown DR, Kurman RJ, Ronnett BM, Stoler MH, Ferenczy A, Harper DM, Tamms GM, Yu J, Lupinacci L, Railkar R, Taddeo FJ, Jansen KU, Esser MT, Sings HL, Saah AJ, Barr E: Prophylactic quadrivalent human papillomavirus (types 6, 11, 16, and 18) L1 virus-like particle vaccine in young women: a randomised double-blind placebo-controlled multicentre phase II efficacy trial. Lancet Oncol 2005, 6(5):271-278.

6. Arbyn M, Dillner J: Review of current knowledge on HPV vaccination: an appendix to the European guidelines for quality assurance in cervical cancer screening. J Clin Virol 2007, 38(3):189-197.
7. Ault KA: Effect of prophylactic human papillomavirus L1 virus-likeparticle vaccine on risk of cervical intraepithelial neoplasia grade 2, grade 3, and adenocarcinoma in situ: a combined analysis of four randomised clinical trials. Lancet 2007, 369(9576):1861-1868.

8. Popat S, Hughes S, Papadopoulos P, Wilkins A, Moore S, Priest K, Meehan L, Norton A, O'Brien M: Recurrent responses to non-small cell lung cancer brain metastases with erlotinib. Lung Cancer 2007, 56(1):135-137.

9. Regan DG, Philp DJ, Hocking JS, Law MG: Modelling the population-level impact of vaccination on the transmission of human papillomavirus type 16 in Australia. Sex Health 2007, 4(3):147-163.

10. Mandic A, Vujkov T: Human papillomavirus vaccine as a new way of preventing cervical cancer: a dream or the future? Ann Oncol 2004, 15(2):197-200.

11. Smith JS, Lindsay L, Hoots B, Keys J, Franceschi S, Winer R, Clifford GM: Human papillomavirus type distribution in invasive cervical cancer and high-grade cervical lesions: a meta-analysis update. Int J Cancer 2007, 121(3):621-632.

12. Munger K, Baldwin A, Edwards KM, Hayakawa H, Nguyen CL, Owens M, Grace M, Huh K: Mechanisms of human papillomavirus-induced oncogenesis. J Virol 2004, 78(21):11451-11460.

13. Roden RB, Ling M, Wu TC: Vaccination to prevent and treat cervical cancer. Hum Pathol 2004, 35(8):971-982.

14. Samara RN, Khleif SN: HPV as a model for the development of prophylactic and therapeutic cancer vaccines. Curr Mol Med 2009, 9(6):766-773.

15. Gilboa E: DC-based cancer vaccines. J Clin Invest 2007, 117(5):1195-1203.

16. Bubenik J: Genetically engineered dendritic cell-based cancer vaccines (review). Int J Oncol 2001, 18(3):475-478.

17. Rahma OE, Ashtar E, Czystowska M, Szajnik ME, Wieckowski E, Bernstein S, Herrin VE, Shams MA, Steinberg SM, Merino M, Gooding W, Visus C, Deleo AB, Wolf JK, Bell JG, Berzofsky JA, Whiteside TL, Khleif SN: A gynecologic oncology group phase II trial of two p53 peptide vaccine approaches: subcutaneous injection and intravenous pulsed dendritic cells in high recurrence risk ovarian cancer patients. Cancer Immunol Immunother 2012, 61(3):373-84.

18. Schuler G, Steinman RM: Dendritic cells as adjuvants for immunemediated resistance to tumors. J Exp Med 1997, 186(8):1183-1187.

19. Schuler G, Schuler-Thurner B, Steinman RM: The use of dendritic cells in cancer immunotherapy. Curr Opin Immunol 2003, 15(2):138-147.

20. Banchereau J, Steinman RM: Dendritic cells and the control of immunity. Nature 1998, 392(6673):245-252.

21. Ferrara A, Nonn M, Sehr P, Schreckenberger C, Pawlita M, Durst M, Schneider A, Kaufmann AM: Dendritic cell-based tumor vaccine for cervical cancer II: results of a clinical pilot study in 15 individual patients. J Cancer Res Clin Oncol 2003, 129(9):521-530.

22. Santin AD, Bellone S, Palmieri M, Ravaggi A, Romani C, Tassi R, Roman JJ, Burnett A, Pecorelli S, Cannon MJ: HPV16/18 E7-pulsed dendritic cell vaccination in cervical cancer patients with recurrent disease refractory to standard treatment modalities. Gynecol Oncol 2006, 100(3):469-478.

23. Borysiewicz LK, Fiander A, Nimako M, Man S, Wilkinson GW, Westmoreland D, Evans AS, Adams M, Stacey SN, Boursnell ME, Rutherford E, Hickling JK, Inglis SC: A recombinant vaccinia virus encoding human papillomavirus types 16 and 18, E6 and E7 proteins as immunotherapy for cervical cancer. Lancet 1996, 347(9014):1523-1527.

24. Kenter GG, Welters MJ, Valentijn AR, Lowik MJ, Berends-van der Meer DM, Vloon AP, Essahsah F, Fathers LM, Offringa R, Drijfhout JW, Wafelman AR, Oostendorp J, Fleuren GJ, van der Burg SH, Melief CJ: Vaccination against HPV-16 oncoproteins for vulvar intraepithelial neoplasia. N Engl J Med 2009, 361(19):1838-1847.

doi:10.1186/s12967-014-0353-4

Cite this article as: Rahma et al:: Pre-immature dendritic cells (PIDC) pulsed with HPV16 E6 or E7 peptide are capable of eliciting specific immune response in patients with advanced cervical cancer. Journal of Translational Medicine 2014 12:353. 